\title{
Sonda uretral flexível como método alternativo para aferição invasiva da pressão intracraniana em trauma cranioencefálico induzido em coelhos
}

\author{
[Flexible urethral catheter as alternative method to invasive measurement of intracranial \\ pressure in induced head trauma in rabbits] \\ G. Aiello ${ }^{1}$, A.O. Andrades ${ }^{1}$, A. Ripplinger ${ }^{1}$, A.V. Soares ${ }^{2}$, D. Polidoro ${ }^{1}$, M.A.B. Vaz $^{1}$, \\ A.C.Colvero ${ }^{3}$, R.P. Santos ${ }^{1}$, R. Conceição ${ }^{3}$, R.O. Chaves $^{1}$, A. Mazzanti ${ }^{2 *}$ \\ ${ }^{1}$ Aluno de pós-graduação - Universidade Federal de Santa Maria - UFSM - Santa Maria, RS \\ ${ }^{2}$ Universidade Federal de Santa Maria - UFSM - Santa Maria, RS \\ ${ }^{3}$ Aluno de graduação - Universidade Federal de Santa Maria - UFSM - Santa Maria, RS
}

\begin{abstract}
RESUMO
O objetivo deste estudo foi utilizar a sonda uretral flexível como método alternativo para aferição da pressão intracraniana em coelhos com trauma cranioencefálico induzido pelo cateter de Fogarty $4 \mathrm{Fr}$ (balão epidural) e comparar os dados obtidos com o método convencional de cateter de ventriculostomia. Foram utilizados 12 coelhos, machos, adultos, distribuídos aleatoriamente em dois grupos, denominados de G1: mensuração da PIC com cateter de ventriculostomia $(n=6)$ e $\mathrm{G} 2$ : mensuração com sonda uretral $(\mathrm{n}=6)$. Foram realizadas duas craniotomias na região parietal direita e esquerda para a implantação do cateter de ventriculostomia ou sonda uretral flexível e o balão epidural, respectivamente. A PAM, a PPC, a FC, a FR e a TR foram mensurados antes e após a craniotomia. A PIC foi avaliada após a craniotomia e a cada 10 minutos depois do preenchimento do balonete com $0,3 \mathrm{~mL}$ de $\mathrm{NaCl} 0,9 \%$, durante 40 minutos, e com $0,6 \mathrm{~mL}$, pelo mesmo período de tempo, totalizando 80 minutos. A PIC aumentou em ambos os grupos, sendo menores os valores registrados com a sonda uretral flexível. Foi possível reproduzir o aumento da PIC com o modelo experimental de TCE utilizando o cateter de Fogarty $4 \mathrm{Fr}$ na região epidural e, embora haja a necessidade de outros estudos, a sonda uretral flexível demonstra ser um método alternativo de mensuração da PIC em coelhos com trauma cranioencefálico.
\end{abstract}

Palavras-chave: cateter de ventriculostomia, autorregulação, neurologia, cirurgia

\begin{abstract}
The aim of this study was to evaluate the use of flexible urethral catheter as an alternative method for measuring intracranial pressure in rabbits with head trauma induced by 4 F Fogarty catheter (epidural balloon) and compare the data obtained with the conventional method of ventriculostomy catheter. In this study, New Zealand rabbits were randomly distributed into two groups, G1: measuring the ICP with ventriculostomy catheter $(n=6)$ and G2: measuring the ICP with urethral catheter (n=6). Two craniotomies were performed in the right and left parietal region for the implantation of a ventriculostomy catheter and/or flexible urethral catheter and epidural 4 Fr Fogarty arterial embolectomy catheter, respectively. MAP, CPP, HR, RF and RT values were measured before and after of the craniotomy. The ICP value was measured after craniotomy, every five minutes during 40 minutes after the balloon was inflated with $0.3 \mathrm{ml}$ with $\mathrm{NaCl}$ and further 40 minutes after the balloon was inflated with $0.6 \mathrm{ml}$. The ICP value increased in both groups; however, the ICP values were lower in the flexible urethral catheter. The flexible urethral catheter can be used as an alternative method to measure ICP values in rabbits with head injury.
\end{abstract}

Keywords: ventriculostomy catheter, autoregulation, neurology, surgery

Recebido em 19 de maio de 2016

Aceito em 19 de outubro de 2016

*Autor para correspondência (corresponding author)

E-mail: alexamazza@yahoo.com.br 


\section{INTRODUÇÃO}

O traumatismo cranioencefálico (TCE) está associado à alta taxa de mortalidade e morbidade em humanos e pequenos animais (Dewey e Fletcher, 2016). Pode ser dividido em eventos primários, que ocorrem no momento da injúria, e secundários, provocados pelos processos bioquímicos, inflamatórios e metabólicos (Lorenz et al., 2011).

A mensuração da pressão intracraniana (PIC) é empregada para o diagnóstico da hipertensão intracraniana e também para o valor prognóstico, especialmente nos pacientes com graves sinais clínicos devido ao TCE. Ainda, pode ser utilizada como parâmetro na avaliação das medidas terapêuticas empregadas para reduzir a PIC, sendo imprescindível para determinar os valores da pressão de perfusão cerebral (PPC) (Carlotti et al., 1998).

Para a mensuração da PIC, existem métodos invasivos e não invasivos (Bratton et al., 2007). $\mathrm{O}$ uso de cateter intraventricular é considerado o "padrão ouro" para a monitorização invasiva (Maniker et al., 2006) por permitir a aferição precisa da PIC (Smith, 2008). Existem vários equipamentos utilizados para monitorização intraventricular, entre eles, pode ser citado o aparelho por fibra óptica, considerado de alto custo, e o uso de cateter intraventricular acoplado a um monitor multiparamétrico (Roux, 2013), que, além do menor custo, tem a possibilidade terapêutica de drenagem liquórica (Giugno et al., 2003).

A monitorização da PIC é rotineiramente utilizada em pacientes humanos (Dewey e Fletcher, 2016), porém poucos são os hospitais e as clínicas veterinárias que mensuram a PIC em pacientes com trauma cranioencefálico. Vários são os motivos, entre eles, o custo dos métodos empregados (Packer et al., 2011), sendo necessária a busca por métodos alternativos de fácil aplicação e de baixo custo.

Diante disso, o objetivo deste estudo foi utilizar a sonda uretral flexível como método alternativo para a aferição da pressão intracraniana em coelhos com trauma cranioencefálico induzido por cateter Fogarty $4 \mathrm{Fr}$ (balão epidural) e comparar os dados obtidos com o método convencional de cateter de ventriculostomia.

\section{MATERIAL E MÉTODOS}

Foram utilizados 12 coelhos, machos, da raça Nova Zelândia, entre 3,3-4,0kg de peso vivo, provenientes do Biotério Central da Instituição. Os animais permaneceram alojados em gaiolas individuais, com água e ração comercial ad libitum, por um período de 10 dias antes do início do experimento. Este trabalho foi aprovado pelo Comitê de Ética em Pesquisa da Instituição, sob o número 045/2014.

Os coelhos foram distribuídos aleatoriamente em dois grupos experimentais, com seis indivíduos cada: grupo 1 - G1: avaliação contínua da PIC pelo método do cateter de ventriculostomia (Kit Kompacto, Ventura, Brasil) e grupo 2 - G2: avaliação contínua da PIC pelo método intraventicular por sonda uretral flexível (Mark Med, Brasil). Ambos os dispositivos foram acoplados à um monitor multiparamétrico.

Para realização da craniotomia e acesso ao ventrículo lateral do encéfalo, foi realizada a tricotomia da face externa das orelhas direita e esquerda e do crânio (ossos frontal e parietal). Como medicação pré-anestésica (MPA), foi administrado midazolam (1mg. $\mathrm{kg}^{-1}$, intramuscular) associado à morfina $\left(2 \mathrm{mg} \cdot \mathrm{kg}^{-1}\right.$, intramuscular), 10 minutos antes de acessar a veia marginal da orelha esquerda. Em seguida, os coelhos foram submetidos à anestesia geral, por meio da vaporização de isoflurano na máscara facial, intubados com máscara laríngea e mantidos em anestesia geral inalatória com o mesmo agente anestésico, em vaporizador calibrado na concentração de 1,5 CAM (concentração alveolar mínima).

A artéria auricular direita foi acessada com cateter $22 \mathrm{G}$ acoplado a um transdutor de pressão e este sistema a um monitor multiparamétrico para mensuração da pressão arterial média (PAM). A temperatura corporal foi monitorada utilizando-se uma probe retal e mantida entre 38 e $39^{\circ} \mathrm{C}$ durante todo o procedimento cirúrgico.

Todos os animais foram posicionados em decúbito esternal. Antes da intervenção cirúrgica, foram mensuradas a pressão arterial média (PAM), a frequência cardíaca (FC), a frequência respiratória (FR) e a temperatura retal (TR), três vezes, com intervalo de cinco minutos (denominados de C0, C5 e C10). Foi colhido 
$0,3 \mathrm{~mL}$ de sangue arterial para hemogasometria com início antes da craniotomia e a cada 15 minutos até o término, totalizando oito avaliações.

Para a realização do procedimento cirúrgico, foi realizada a antissepsia com álcool-iodo-álcool e uma incisão longitudinal da pele, do subcutâneo, e do periósteo, sobre a linha média do crânio de, aproximadamente, $5,0 \mathrm{~cm}$. Utilizando-se um elevador de periósteo, iniciou-se o deslocamento dele na região parietal esquerda e direita. Com um motor de suspensão elétrico de baixa rotação acoplado a uma broca sulcada de $3,0 \mathrm{~mm}$ de diâmetro, dois orifícios no crânio foram criados sobre o osso parietal, situados a $2,5 \mathrm{~mm}$ em posição lateral direita e esquerda da sutura sagital e $5 \mathrm{~mm}$ caudal à sutura coronária (Fig. 1A). Através do orifício direito, foi introduzido o cateter de ventriculostomia (G1) ou a sonda uretral flexível número seis (G2), em uma profundidade de $6 \mathrm{~mm}$, compatível com a entrada no interior do ventrículo lateral direito. A confirmação do posicionamento do dispositivo dentro do ventrículo ocorreu ao se visualizar o líquido cerebroespinhal (LCE) fluindo pelo cateter ou pela sonda uretral (Fig. 1B). Para evitar extravasamento de LCE e fixar o cateter ou a sonda uretral, o orifício da craniotomia foi selado com resina acrílica autopolimerizável. Com o cateter de ventriculostomia (G1) ou a sonda uretral flexível (G2) inseridos, e antes de introduzir o balão epidural, foram mensurados novamente a PAM, a FC, a FR, o TPC e a TR, três vezes, com intervalo de cinco minutos (denominados de $\mathrm{Cr} 0$, Cr5 e Cr10). Em seguida, as aferições foram anotadas, juntamente com os valores da PIC basal, em todos os coelhos dos dois grupos.

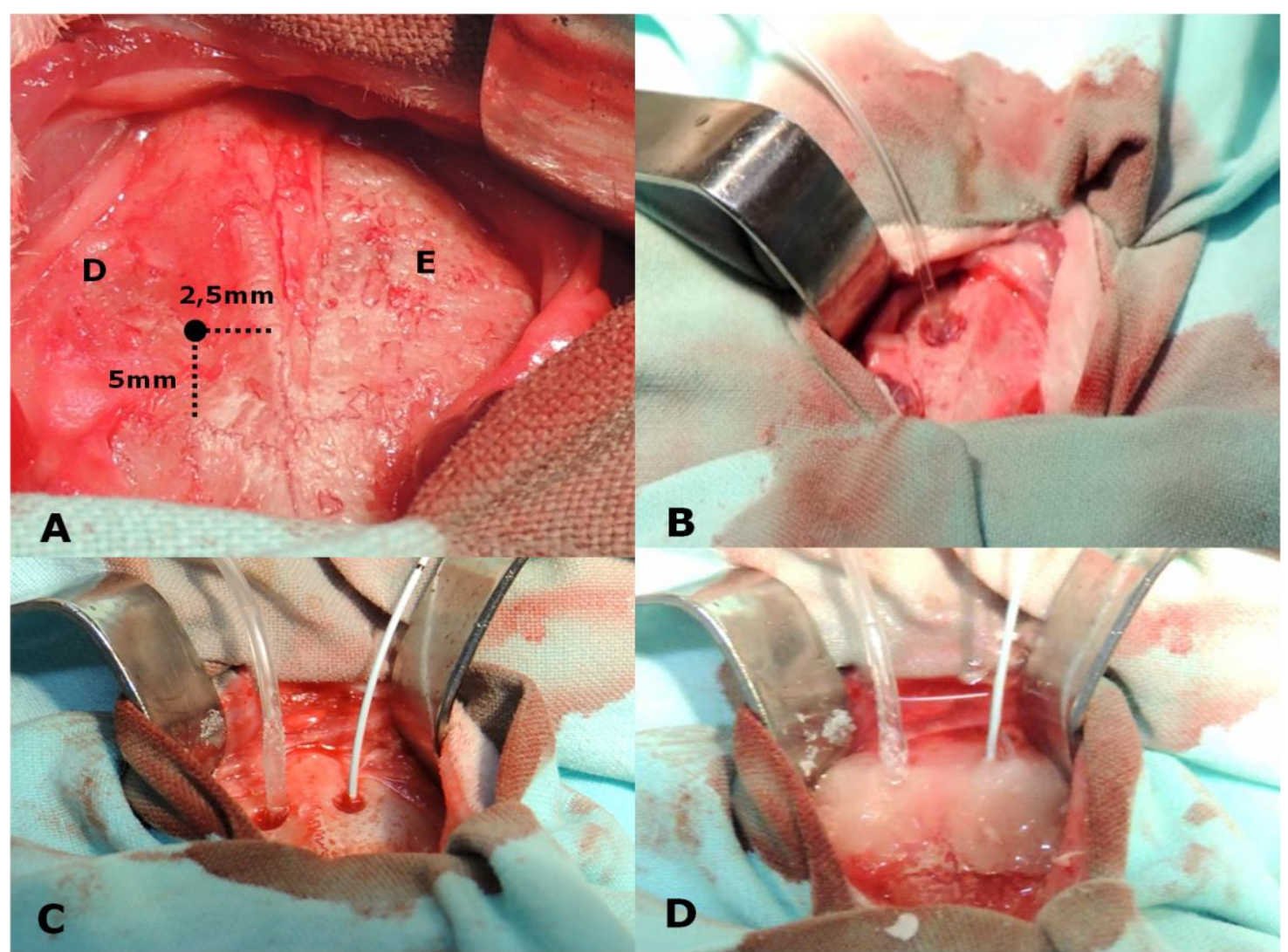

Figura 1. Implantação da sonda uretral flexível intraventricular para aferir a PIC e confecção do modelo experimental de TCE em coelhos. A) Mensurações na região parietal direita para a realização da craniotomia; B) LCE no interior da sonda uretral flexível, confirmando a localização da sonda uretral no ventrículo lateral direito. C) Sonda uretral flexível inserida na craniotomia direita e o cateter de Fogarty 4 $F R$ na esquerda. D) Oclusão das craniotomias e fixação dos cateteres com resina acrílica autopolimerizável. 
Para a realização do modelo experimental de TCE por balão epidural, o cateter de Fogarty 4 Fr (ED - alas Lifesciences LLC Irvine, EUA) foi introduzido no interior do orifício da região parietal esquerda (Fig. 1C), posicionado no espaço epidural (entre o osso do crânio e a duramáter), e foi realizado o selamento hermético com resina acrílica autopolimerizável (Fig. 1D). Na sequência, o balão do cateter de Fogarty 4 Fr foi preenchido com $0,3 \mathrm{~mL}$ de solução salina isotônica $(\mathrm{NaCl}$ a $0,9 \%)$, durante 40 minutos, para mensuração dos valores da pressão intracraniana (PIC), da PAM, da FC, da FR, do TPC, do $\mathrm{CO}_{2}$ e da TR a cada cinco minutos. A PPC foi calculada por meio da fórmula: PPC=PAM-PIC (Dewey e Fletcher, 2016).

Decorridos 40 minutos, o balão foi preenchido com mais $0,3 \mathrm{~mL}$, totalizando $0,6 \mathrm{~mL}$ de $\mathrm{NaCl}$ $0,9 \%$, que permaneceu inflado pelo mesmo período de tempo, finalizando 80 minutos de mensuração, ou seja, 40 minutos com balão epidural preenchido com $0,3 \mathrm{~mL}$ e 40 minutos com $0,6 \mathrm{~mL}$ de $\mathrm{NaCl} 0,9 \%$. Ao término das avaliações, os animais foram submetidos à eutanásia por meio de sobredose anestésica com isoflurano, seguida da administração de cloreto de potássio $(\mathrm{KCl}$ a $10 \%)$ até a interrupção dos batimentos cardíacos. O cateter/sonda uretral flexível e o cateter de Fogarty $4 \mathrm{Fr}$ foram removidos, e os locais de inserção inspecionados em busca de hematomas.

Foi realizada análise estatística pelo modelo de regressão linear clássico, aplicando-se o teste t de Student para verificar a significância dos coeficientes $(\mathrm{P}<0,05)$ para comparar os métodos de aferição da PIC.

\section{RESULTADOS E DISCUSSÃO}

Neste estudo, o modelo experimental de TCE por redução de complacência, utilizando-se um cateter de Fogarty 4 Fr na região epidural, foi de fácil execução, de baixo custo e permitiu elevar a pressão intracraniana. Esse mesmo método foi realizado com êxito por Douzinas et al. (1999) e Çagavi et al. (2005), utilizando-se cateter de Fogarty, e por Abe et al. (1984); Mizumoto et al. (2005), utilizando sonda de Foley.

Dois coelhos foram excluídos das análises dos dados deste estudo (G1 e G2), pois foram a óbito antes do término das mensurações. A PIC máxima observada antes do óbito foi $86 \mathrm{mmHg}$ no $\mathrm{G} 1$ e $78 \mathrm{mmHg}$ no $\mathrm{G} 2$, o que correspondeu sete vezes o valor da PIC normal em coelhos (5 a $13 \mathrm{mmHg}$ ) (Çagavi et al., 2005). Nesses animais, foi realizada a craniotomia descompressiva e verificado deslocamento do cerebelo através do forame magno, caracterizando herniação foraminal. Para Freeman e Platt (2011), esse tipo de herniação é frequentemente fatal, pois causa parada respiratória por compressão dos centros respiratórios na medula oblonga.

As três principais complicações associadas à inserção de cateteres intraventriculares são: hemorragia intracraniana, infecção e obstrução (Li et al., 2010). Binz et al. (2009) observaram complicações hemorrágicas associadas a cateteres intraventriculares em $5,7 \%$ dos pacientes avaliados, porém, estas foram clinicamente significativas em menos de $1 \%$. O hematoma subdural é outra possível complicação (Lang et al., 2012). No presente estudo, não foram observadas complicações associadas à inserção (hemorragia, hematoma e obstrução) em ambos os grupos. No estudo de Çagavi et al. (2005), que também mensurou a PIC intraventricular em coelhos, não foi observada a presença de hematomas.

Os valores de $\mathrm{PaCO}_{2}$ considerados normais para coelhos variam entre 20 e $46 \mathrm{mmHg}$ (Barzago et al., 1992). Neste estudo, todos os animais apresentaram hipercapnia em todos os tempos avaliados, inclusive na avaliação da $\mathrm{PaCO}_{2}$ basal (Fig. 2B). A hipercapnia observada na mensuração basal, antes de se realizar a craniotomia e o modelo experimental de TCE, pode estar relacionada ao tempo entre a indução anestésica utilizando a máscara facial e a introdução da máscara laríngea, à ausência de ventilação mecânica e aos fármacos administrados. A morfina é um $\mu$ agonista, que produz analgesia, euforia e uma importante depressão respiratória em coelhos (Donnelly e Warren, 2014). Vários estudos mostram que os benzodiapínicos, como o midazolam, causam média a moderada hipercapnia em coelhos e humanos. (Forster et al., 1980; Chang et al., 2009). Porém, no estudo de Schroeder e Smith (2011), os quais avaliaram a taxa respiratória e realizaram as análises de gases em coelhos medicados com opioides (buprenorfina, butorfanol), midazolam e a associação do midazolam aos opioides isoladamente, não foi 
evidenciada hipercapnia. Neste estudo, não houve diferença estatística entre os tempos avaliados e entre os grupos. Mesmo não havendo diferença estatística entre os tempos, houve uma tendência de aumento crescente dos valores da $\mathrm{PaCO}_{2}$, após se iniciar o modelo experimental de TCE.

A $\mathrm{PaO}_{2}$ demonstra o balanço entre a quantidade de oxigênio fornecida pela ventilação e o quanto é transferido dos alvéolos para os capilares (Amaral et al., 1992). O valor estimando da $\mathrm{PaO}_{2}$ em coelhos respirando ar ambiente é $90 \mathrm{mmHg}$ (Barzago et al., 1992). Neste estudo, todos os coelhos apresentaram valores superiores ao preconizado como normal para a espécie. Não houve diferença estatística entre os grupos e entre os tempos, exceto na última avaliação (35minutos de avaliação, após preencher o balonete com $0,6 \mathrm{~mL}$ de solução salina) no grupo que mensurou a PIC com cateter de ventriculostomia.

$\mathrm{O}$ valor médio do $\mathrm{pH}$ de sangue arterial em coelhos não anestesiados varia entre 7,2 - 7,5 (Donnelly e Warren, 2014). No estudo de Benato et al. (2013), o valor médio estimado do $\mathrm{pH}$ em coelhos anestesiados foi de 7,33 $( \pm 0,07)$, sendo utilizado fentanil, midazolam e isoflurano. Neste estudo, o valor estimado do $\mathrm{pH}$ não apresentou diferença entre os grupos. $\mathrm{O} \mathrm{pH}$ manteve-se dentro do intervalo de normalidade antes e após a craniotomia e até 15 minutos após preencher o balonete com $0,3 \mathrm{~mL}$ de solução salina. Porém, após esse período de avaliação, foi evidenciada acidose em ambos os grupos até a finalização das mensurações.

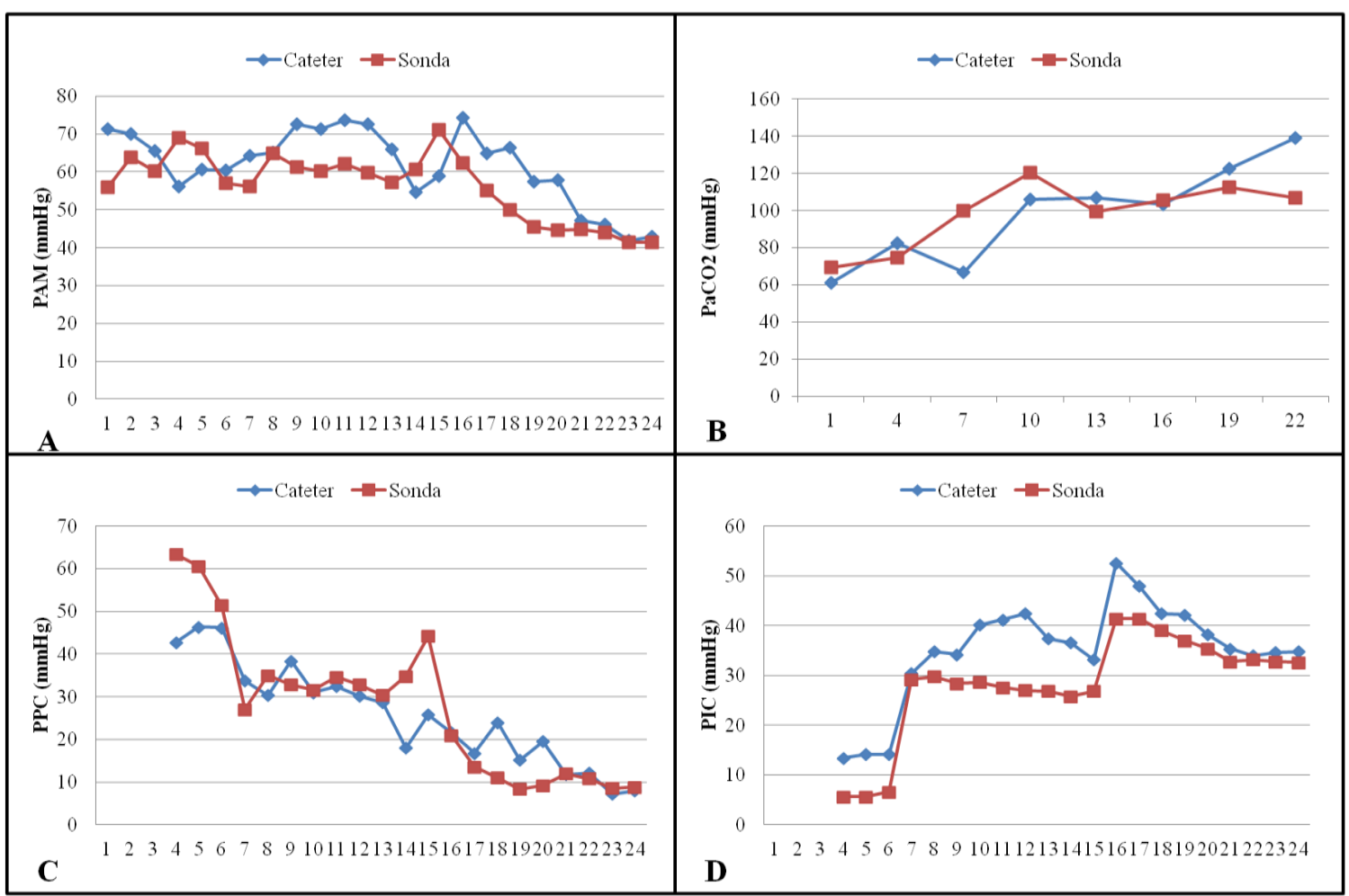

Figura 2. Mensurações da PAM dos grupos cateter de ventriculostomia e sonda uretral (A), do $\mathrm{PaCO}_{2}(\mathrm{~B})$, da PPC (C) e da PIC (D) em coelhos submetidos ao modelo experimental de TCE, utilizando-se o cateter de Fogarty 4 Fr. Nota-se que a PIC registrada com a sonda uretral flexível foi menor e acompanhou as variações da pressão registradas em todos os momentos pelo cateter de ventriculostomia.

Os valores do bicarbonato $\left(\mathrm{HCO}_{3}\right)$ estavam dentro do intervalo de normalidade $(22,37$ a $35,08)$ nos dois grupos avaliados, e não houve diferença significativa entre os tempos. Portanto, o tipo de acidose observado nos coelhos deste estudo, após os 15minutos de avaliação do preenchimento do balonete com $0,3 \mathrm{~mL}$ de solução salina, foi a respiratória, pois foi 
observada hipercapnia nos coelhos de ambos os grupos e normalidade nos valores do bicarbonato.

A autorregulação da PIC é a ocorrência de mecanismos para manter constantes os componentes da cavidade craniana (parênquima encefálico, LCE e sangue) e controlar o FSC quando a PAM estiver entre $50-150 \mathrm{mmHg}$ (Dewey e Fletcher, 2016). Outro mecanismo para a autorregulação da PIC é a química (Carlotti et al., 1998), que se baseia na capacidade de resposta direta da vasculatura cerebral quando houver variação na pressão parcial de dióxido de carbono no sangue arterial $\left(\mathrm{PaCO}_{2}\right)$. Os níveis elevados de $\mathrm{PaCO}_{2}$ causam vasodilatação cerebral, enquanto a diminuição dos níveis de $\mathrm{PaCO}_{2}$ causa vasoconstrição cerebral (Dewey e Fletcher, 2016). Neste estudo, provavelmente ocorreram mecanismos de autorregulação (pressão e química), pois a PAM variou dentro dos valores limites para a sua realização $(41,4$ e $73 \mathrm{mmHg}$ ), bem como os valores da $\mathrm{PaCO}_{2}$ (Fig. 2B), que permaneceram altos durante o preenchimento do balonete, com 0,3 e $0,6 \mathrm{~mL}$, ao serem comparados com o tempo antes da craniotomia, condição para a ativação da autorregulação química.

Quanto à PAM, houve diferença estatística entre os tempos e entre os métodos de mensuração nos tempos $(\mathrm{P}<0,01)$. Após o preenchimento do balonete com $0,3 \mathrm{~mL}$ de $\mathrm{NaCl}$, a PAM aumentou em ambos os grupos, porém os valores em G1 foram maiores que em G2. Após preencher o balonete com $0,6 \mathrm{~mL}$, a PAM aumentou nos dois grupos e os valores do G1 continuaram superiores ao G2. Após cinco minutos de avaliação, a PAM voltou a diminuir até que os valores do G1 e G2 se igualaram. É interessante observar que, mesmo com o aumento da PIC no momento do preenchimento do balonete com 0,3mL, a PAM aumentou somente com cinco minutos de compressão, persistindo a elevação até o $25^{\circ}$ minuto (Fig. 2A). A provável explicação da não elevação da PAM nos primeiros cinco minutos se deve à doutrina de Monro-Kellie, cujo deslocamento do LCE no sentido caudal em direção ao canal central da medula espinhal, bem como a redução na produção, pode auxiliar na manutenção da pressão intracraniana nos momentos iniciais de aumento da PIC (Freeman e Platt, 2011).

A pressão de perfusão cerebral (PPC) em coelhos varia de 85 a $117 \mathrm{mmHg}$ (Çagavi et al., 2005). Neste estudo, foi verificado um valor médio de $29,84 \mathrm{mmHg}$ no $\mathrm{G} 1 \mathrm{e}$ de $33,69 \mathrm{mmHg}$ no G2 quando o balonete foi preenchido com $0,3 \mathrm{~mL} \mathrm{e}$ $15,15 \mathrm{mmHg}$ no $\mathrm{G} 1 \mathrm{e} 11,49 \mathrm{mmHg}$ no $\mathrm{G} 2 \mathrm{com}$ $0,6 \mathrm{~mL}$ de $\mathrm{NaCl} 0,9 \%$ (Fig. 2C). Esse resultado demonstrou que $\mathrm{o}$ aumento da pressão intracraniana no decorrer do tempo provocou a redução da PPC, provavelmente pela diminuição do FSC (Fig. 3). Tal fato indica que, mesmo com os prováveis mecanismos autorregulatórios da PIC presentes, a pressão de perfusão cerebral se manteve baixa e não evitou uma possível isquemia do tecido encefálico (Çagavi et al., 2005)

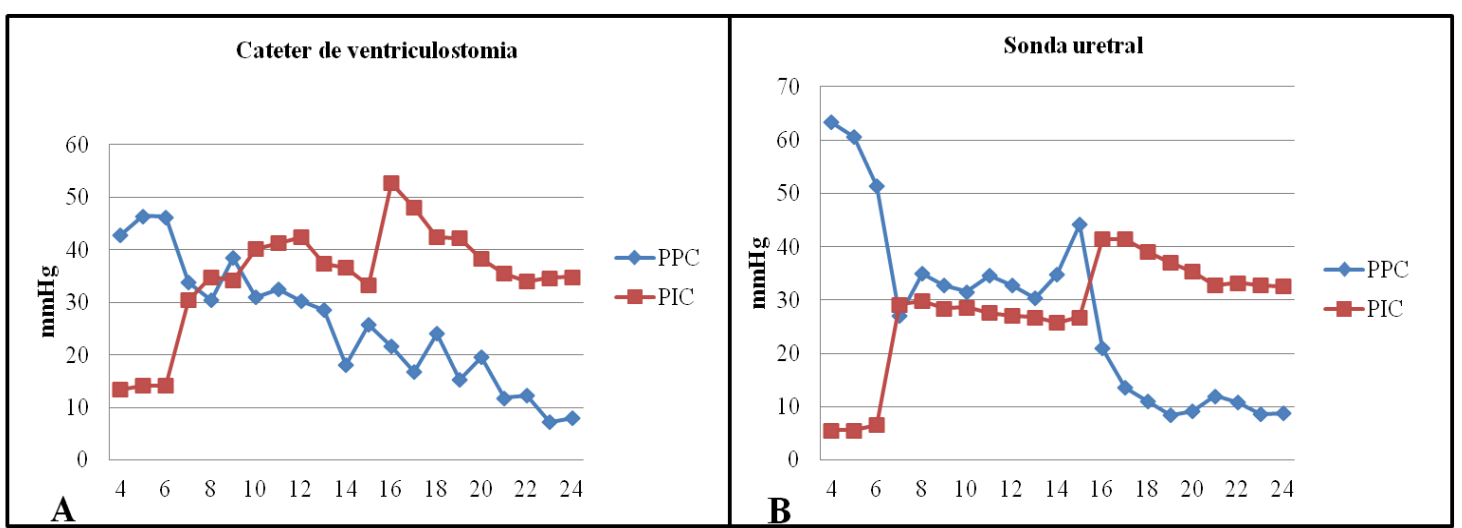

Figura 3. Relação da pressão de perfusão cerebral (PPC) e da pressão intracraniana (PIC) nos grupos cateter de ventriculostomia (A) e sonda uretral flexível (B), em coelhos submetidos ao modelo experimental de TCE, utilizando-se o cateter de Fogarty 4 Fr. Nota-se a diminuição da PPC, conforme o aumento da PIC no grupo com cateter de ventriculostomia após o início do preenchimento do balão com $0,3 \mathrm{~mL}$ de $\mathrm{NaCl} 0,9 \%$. 
As médias estimadas dos valores basais da PIC, ou seja, imediatamente após a craniotomia e decorridos cinco e 10 minutos antes do preenchimento do balão epidural, apresentaram diferença significativa $(\mathrm{P}<0,01)$ entre os grupos (G1: $13,93 \mathrm{mmHg}$ e em $\mathrm{G} 2: 5,93 \mathrm{mmHg}$ ), porém não houve variação entre os tempos em ambos os grupos (Fig. 2D). No estudo de Çagavi et al. (2005), também foi utilizado o cateter intraventricular em coelhos e foi obtido o valor médio da PIC basal intraventricular de $12,7 \mathrm{mmHg}$

Ao preencher o balonete do cateter de Fogarty 4 Fr com $0,3 \mathrm{~mL}$ de solução salina, a PIC em ambos os grupos aumentou significativamente comparada aos valores da PIC basal (Fig. 2D). $\mathrm{O}$ valor médio estimado da PIC nos 40 minutos de avaliação foi de $36,71 \mathrm{mmHg}$ no G1 e de $27,77 \mathrm{mmHg}$ no $\mathrm{G} 2$. Em todo o tempo de avaliação, os valores estimados da PIC mensurados pelo cateter foram significativamente $(\mathrm{P}<0,01)$ superiores aos da sonda uretral flexível. Quando o balonete foi preenchido com $0,6 \mathrm{~mL}$ de $\mathrm{NaCl}$, os valores da PIC foram significativamente maiores do que os valores da PIC basal e quando comparados ao preenchimento com $0,3 \mathrm{~mL}(\mathrm{P}<0,05)$ (Fig. 2D). O valor médio da PIC nos 40 minutos finais de avaliação foi de $39,68 \mathrm{mmHg}$ no $\mathrm{G} 1$ e de $36,68 \mathrm{mmHg}$ no $\mathrm{G} 2$ e foi estatisticamente superior $(\mathrm{P}<0,05)$ aos valores da sonda uretral flexível até 15 minutos de avaliação, não diferindo a partir dos 20 minutos.

Tanto o cateter de ventriculostomia como a sonda uretral flexível são materiais cilíndricos com diâmetros internos similares e com dois orifícios drenantes que se situavam dentro do ventrículo lateral do encéfalo dos coelhos para aferição da PIC. O princípio de mensuração da PIC pelo método intraventricular ocorre pelo deslocamento da coluna líquida e do grau de deformação da parede do cateter, que contribui para o deslocamento do LCE e resulta na leitura de um valor que é registrado pelo monitor multiparamétrico (Srinivasan et al., 2014). Acredita-se que a maneira de aferição da PIC com a utilização da sonda uretral flexível seja a mesma do cateter de ventriculostomia. Por outro lado, a diferença no comprimento entre os cateteres pode ter influenciado na mensuração da
PIC e nas variações dos valores obtidos entre eles. Assim, recomendam-se novas pesquisas para comprovação dessa hipótese acima mencionada.

Mesmo havendo diferença nos valores da PIC entre as duas formas de mensurações, cabe salientar que a variação dos valores ocorreu em ambos os métodos, obtendo-se, com isso, uma relação linear aparente. Assim, foi possível determinar, com o auxílio do teste de regressão, uma diferença da PIC aferida com o cateter de ventriculostomia em relação à sonda uretral flexível, obtendo-se a fórmula: PIC cateter ventriculostomia $=11,43297+0,83634 \times$ PICsonda, cuja precisão é em torno de $54,87 \%$. Outra fórmula obtida com precisão de $86,13 \%$, nos testes estatísticos foi $\mathrm{PIC}=0,001241+$ 0,01661.sonda ${ }^{2}+$ FR.(-9,023+0,01741.FR) + FC. $(-4,882+0,002305 . F C+0,01115$.sonda $+\quad 0,00002452 . \mathrm{FR})+$ oximetria. $(-1,102$ $-0,02606$. Sonda $+0,1666$.horas $+0,06586 . \mathrm{FR}+$ 0,03754.FC - $0,01395 . \mathrm{CO} 2)+$ CO2.(0,003611.CO2 - 0,009116.sonda + 0,02059.horas + 0,01034.FR + 0,002975.FC) + horas.(-7,155 - 0,1777.horas + 0,05662.FR 0,01978.FC), porém com mais limitações em se aplicar por depender de outras variáveis.

Com os resultados obtidos neste estudo, há possibilidade da utilização da sonda uretral flexível em coelhos, como alternativa ao cateter de ventriculostomia disponível comercialmente, cujo custo é extremamente elevado (R\$1300,00) quando comparado ao método empregando a sonda uretral flexível $(\mathrm{R} \$ 0,89)$. Porém, mesmo não fornecendo os valores exatos da PIC em comparação com o cateter de ventriculostomia e, portanto, havendo a necessidade de aplicação de uma fórmula de correção, a relevância deste estudo foi observar que a sonda uretral flexível foi capaz de mensurar as oscilações da PIC durante a monitoração do paciente. Esta etapa é considerada essencial na determinação da evolução do quadro clínico e na resposta terapêutica empregada. Esse achado corrobora os de Czosnyka et al. (2007), ao mencionarem que o valor numérico da PIC não é o dado mais importante obtido durante a monitorização, mas sim a dinâmica da PIC e as formas das ondas, pois, com estes, podem-se obter informações importantes sobre a homeostase cerebral. 


\section{CONCLUSÃO}

Conclui-se que a sonda uretral flexível é de fácil aplicação, de baixo custo e pode ser considerada um material alternativo para a mensuração da PIC intraventricular em coelhos. Porém, os valores mensurados são inferiores aos encontrados pelo cateter de ventriculostomia, necessitando da aplicação de uma fórmula de correção, caso se queira obter os valores exatos da PIC.

\section{REERÊNCIAS}

ABE, T.; BLACK, P.M.; FOLEY, L. Changes in parenchymal and ventricular pressure with experimental epidural compression. Surg. Neurol., v.22, p.477-480, 1984.

AMARAL, J.L.G.; FERREIRA, A.C.P.; FEREZ, D.; GERETTO, P. Monitorização da respiração: oximetria e capnografia. Rev. Bras. Anestesiol., v.42, p.51-58, 1992.

BARZAGO, M.M.; BORTOLOTTI, A.; OMARINI, D. et al. Monitoring of blood gas parameters and acid-base balance of pregnant and non-pregnant rabbits (Oryctolaguscuniculus) in routine experimental conditions. Lab. Anim., v.26, p.73-79, 1992.

BENATO, L.; CHESNEL, M.; EATWELL, K.; MEREDITH, A. Arterial blood gas parameters in pet rabbits anaesthetized using a combination of fentanyl-fluanisonemidazolam-isofluorane. $J$. Small Anim. Pract., v.54, p.343-346, 2013.

BINZ, D.D.; TOUSSAINT, L.G.; FRIEDMAN, J.A. Hemorrhagic complications of ventriculostomy placement: a meta-analysis. Neurocrit. Care, v.10, p.253-256, 2009.

BRATTON, S.L.; BULLOCK, M.R.; CARNEY, $\mathrm{N}$. et al. Guidelines for the management of severe traumatic brain injury. J. Neurotrauma, v.24, p.S37-S44, 2007.

ÇAGAVI, F.; KALAVCI, M.; OZER, Y. et al. Dispersion of cerebral temperature, cerebral perfusion and intracranial pressure in rabbits placed with epidural balloons. Brain Res. Bull., v.64, p.481-485, 2005.

CARLOTTI JR, C.G.; COLLI, B.O.; DIAS, L.A.A. Hipertensão intracraniana. Rev. Med., v.31, p.552-562, 1998.
CHANG, C.; UCHIYAMA, A.M.A.L.; MAHIMO, T.; FUJINO, Y. A comparison of the effects of respiratory carbon dioxide response, arterial blood pressure, and heart rate of dexmedetomidine, propofol, and midazolam in sevoflurane-anesthetized rabbits. Anesth. Analg., v. 109, p.84-89, 2009.

CZOSNYKA, M.; SMIELEWSKI, P.; TIMOFEEV, I. et al. Intracranial pressure: more than a number. Neurosurg. Focus, v.22, p.E10, 2007.

DEWEY, C.W.; FLETCHER, D.J. Head-trauma management. In: DEWEY, C.W.; COSTA, R.C. (Eds.). Practical guide to canine and feline neurology. Ames: JohnWiley \& Sons, 2016. p.237-248.

DONNELLY, T.M.; WARREN, K.S. Textbook of rabbit medicine. London: Elsevier, 2014. p.187-248.

DOUZINAS, E.E.; KOSTOPOULOS, V.; KYPRIADES, E. et al. Brain eigen frequency shifting as a sensitive index of cerebral compliance in an experimental model of epidural hematoma in the rabbit: preliminary study. Crit. Care. Med., v.27, p.978-984, 1999.

FORSTER, A.; GARDAZ, J.P.; SUTER, P.M.; GEMPERLE, M. Respiratory depressin by midazolam and diazepam. Anesthesiology, v.53, p.494-497, 1980.

FREEMAN, A.C.; PLATT, S.R. Head trauma. In: PLATT, S.R.; GAROSI, L.S. (Eds.). Small animal neurological emergencies. London: Manson, 2011. p.323-382.

GIUGNO, K.M.; MAIA, T.R.; KUNRATH, C.L.; BIZZI, J.J. Tratamento da hipertensão intracraniana. J. Pediat., v.79, p.287-96, 2003.

LANG, S.S.; KOFKE, W.A.; STIEFEL, M.F. Monitoring and intraoperative management of elevated intracranial pressure and decompressive craniectomy. Anesthesiol. Clin., v.30, 289-310, 2012.

LI, L.M.; TIMOFEEV, I.; CZOSNYKA, M.; HUTCHINSON, P.J. Review article: the surgical approach to the management of increased intracranial pressure after traumatic brain injury. Anesth. Analg., v.111, p.736-748, 2010. 
LORENZ, M.D.; COATES, J.R.; KENT, M. Stupor or coma. In: LORENZ, M.D.; DACVIM, D.V.M.; COATES, J. Handbook of veterinary neurology. 5.ed. [Amsterdã]: Elsevier, 2011. p.346-383.

MANIKER, A.H.; VAYNMAN, A.Y.; KARIMI, R.J. et al. Hemorrhagic complications of external ventricular drainage. Neurosurgery, v.59, p.419424, 2006.

MIZUMOTO, N.; TANGO, H.K.; PAGNOCCA, M.L. Efeitos da hipertensão arterial induzida sobre a complacência e pressão de perfusão encefálica em hipertensão intracraniana experimental: comparação entre lesão encefálica criogênica e balão subdural. Rev. Bras. Anestesiol., v.55, p.289-298, 2005.

PACKER, R.A.; SIMMONS, J.P.; DAVIS, N.M.; CONSTABLE, P.D. Evaluation of an acute focal epidural mass model to characterize the intracranial pressure-volume relationship in healthy Beagles. Am. J. Vet. Res., v.72, p.103-8, 2011.
ROUX, P.L. Physiological Monitoring of the severe traumatic brain injury patient in the intensive care unit. Cur. Neurol. Neurosci. Rep., v.13, p.1-16, 2013.

SCHROEDER, C.A.; SMITH, L.J. Respiratory rates and arterial blood gas tensions in healthy rabbits given buprenorphine, butorphanol, midazolam, or their combinations. J. Am. Assoc. Lab. Anim. Sci., v.50, p.205-2011, 2011.

SMITH, M. Monitoring intracranial pressure in traumatic brain injury. Anesth. Analg., v.106, p.240-248, 2008.

SRINIVASAN, V.M.; O`NEILL, B.R.; JHO, D. et al. The history of external ventricular drainage. Neurosurgery, v.120, p.228-236, 2014. 